\title{
Self-reported face recognition is highly valid, but alone is not highly discriminative of prosopagnosia-level performance on objective assessments
}

\author{
Joseph M. Arizpe ${ }^{1,2,3}$ (D) Elyana Saad ${ }^{1,2} \cdot$ Ayooluwa O. Douglas $^{1,2} \cdot$ Laura Germine $^{1,4} \cdot$ Jeremy B. Wilmer $^{5}$. \\ Joseph M. DeGutis ${ }^{1,2}$
}

Published online: 13 February 2019

(C) The Psychonomic Society, Inc. 2019

\begin{abstract}
Severe developmental deficits in face recognition ability (developmental prosopagnosia, or DP) have been vigorously studied over the past decade, yet many questions remain unanswered about their origins, nature, and social consequences. A rate-limiting factor in answering such questions is the challenge of recruiting rare DP participants. Although self-reported experiences have long played a role in efforts to identify DPs, much remains unknown about how such self-reports can or should contribute to screening or diagnosis. Here, in a large, population-based web sample, we investigated the effectiveness of self-report, used on its own, as a screen to identify individuals who will ultimately fail, at a conventional cutoff, the two types of objective tests that are most commonly used to confirm DP diagnoses: the Cambridge Face Memory Test (CFMT) and the famous faces memory test (FFMT). We used a highly reliable questionnaire (alpha = .91), the Cambridge Face Memory Questionnaire $($ CFMQ), and revealed strong validity via high correlations of .44 with the CFMT and .52 with the FFMT. However, cutoff analyses revealed that no CFMQ score yielded a clinical-grade combination of sensitivity and positive predictive value in enough individuals to support using it alone as a DP diagnostic or screening tool. This result was replicated in an analysis of data from the widely used PI20 questionnaire, a 20-question self-assessment of facial recognition similar in form to the CFMQ. We therefore recommend that screens for DP should, wherever possible, include objective as well as subjective assessment tools.
\end{abstract}

Keywords Self-report $\cdot$ Ability $\cdot$ Individual differences $\cdot$ Face recognition $\cdot$ Screening $\cdot$ Prosopagnosia $\cdot$ Meta-cognition

Over the last 20 years, interest has been growing in identifying and studying individuals with poor face recognition abilities, especially those with developmental prosopagnosia.

Electronic supplementary material The online version of this article (https://doi.org/10.3758/s13428-018-01195-w) contains supplementary material, which is available to authorized users.

Joseph M. Arizpe

Joseph.Arizpe.10@alumni.ucl.ac.uk

1 Department of Psychiatry, Harvard Medical School, Boston, MA, USA

2 Boston Attention and Learning Laboratory, VA Boston Healthcare System, Boston, MA, USA

3 Science Applications International Corporation (SAIC), Fort Sam Houston, TX, USA

4 Institute for Technology in Psychiatry, McLean Hospital, Belmont, MA, USA

5 Department of Psychology, Wellesley College, Wellesley, MA, USA
Developmental prosopagnosia (DP) is characterized as a facial recognition impairment, believed to have been present throughout one's life, that is not due to some more general deficit or organic cause (e.g., poor low-level vision or earlylife brain trauma). It has been suggested as having a prevalence of approximately $2.5 \%$ of the general population (Bowles et al., 2009; Kennerknecht et al., 2006; Kennerknecht, Nga, \& Wong, 2008a), though it should be noted that this estimate is based solely on self-report data and on a common rule of thumb for determining statistical outliers (Barton \& Corrow, 2016). Researchers have been intent on developing a better understanding of the cognitive and neural mechanisms of prosopagnosia, and correspondingly have sought new methods to screen the population to find, and potentially aid in the diagnosis of, research participants with prosopagnosia. Effective screening is necessary to avoid the cost (in time, personnel, resources, and travel) involved in having to assess a preponderance of persons without prosopagnosia in order to obtain only a small handful who do have prosopagnosia. Conversely, efficiently screening out 
persons with prosopagnosia from studies of normal face processing is not widely performed and would be quite useful. Additionally, there are many real-world applications for which efficient identification of individuals with poor facial recognition abilities would be of value. For example, employers may want to avoid placing individuals with below-average face recognition abilities in positions that require sensitive facematching tasks as part of their job (e.g., passport officers; White, Kemp, Jenkins, Matheson, \& Burton, 2014).

One potential method of screening for facial recognition deficits is to use a self-administered questionnaire analogous to that used to screen for developmental disorders such as dyslexia. Scores on the Adult Reading Questionnaire along with a dyslexia status question (i.e., "Do you think you are dyslexic?") have been reported to enable the detection of $62.5 \%$ of those with poor reading skills (putative dyslexia), as determined by objective testing, and the correct rejection of $95.04 \%$ of those without (Snowling, Dawes, Nash, \& Hulme, 2012). In an attempt to efficiently identify and study individuals with poor facial recognition abilities in a similar manner, several research groups have developed self-reported facial recognition ability questionnaires or clinical interviews, the scores of which have been reported to be associated with other measures of facial recognition performance (De Heering \& Maurer, 2014; Kennerknecht et al., 2006; Kennerknecht, Plümpe, Edwards, \& Raman, 2007; Palermo et al., 2017; Shah, Gaule, Sowden, Bird, \& Cook, 2015a; Turano, Marzi, \& Viggiano, 2016). Prior studies have reported moderate-tostrong correlations between self-reported face recognition abilities and objective performance, for typical participants (Bindemann, Attard, \& Johnston, 2014; Gray, Bird, \& Cook, 2017; Livingston \& Shah, 2017; Rotshtein, Geng, Driver, \& Dolan, 2007; Shah, Gaule, Sowden, Bird, \& Cook, 2015a; Shah, Sowden, Gaule, Catmur, \& Bird, 2015b; Turano et al., 2016; Turano \& Viggiano, 2017; Wilmer et al., 2010; though see Palermo et al., 2017) as well as for those with developmental prosopagnosia (Livingston \& Shah, 2017; Stollhoff, Jost, Elze, \& Kennerknecht, 2011). The reported correlation magnitudes are usually in the range of .35 to .55 . This relationship is modulated somewhat by whether the questionnaire is administered before or after the objective assessments, especially if feedback is given, thus highlighting the importance of administering questionnaires while participants are still naive to the objective tests (Bobak, Mileva, \& Hancock, 2018). This apparent relation between self-report and objective facial recognition ability suggests that there may be utility in using self-report as a tool for predicting whether one has prosopagnosia, as defined by objective testing.

Though such self-report questionnaires and clinical interviews for prosopagnosia seem to show promise in terms of their validity as indices of facial recognition, it is unclear whether a score cutoff could be defined for any such self-report measure that would be both highly predictive of and highly sensitive to prosopagnosia, as determined by objective tests of facial recognition. Therefore, in the present study, we sought to assess the utility of the Cambridge Face Memory Questionnaire (CFMQ, a selfreport measure similar to those from other research groups) to screen for prosopagnosia, using a large online data set $(N=1,518)$ containing a diverse sample of participants drawn from the general population. In accord with the current standard of objective measurement for face recognition ability, we also collected performance scores on the Cambridge Face Memory Test (CFMT) and a famous faces memory test (FFMT) for each participant. Because there is also publicly available normative data on the PI20 (Gray et al., 2017; Shah, Gaule, et al., 2015a), another self-report questionnaire of facial recognition ability similar to the CFMQ, it was possible to likewise assess the utility of the PI20 as a screen for prosopagnosia.

We first sought to establish that the CFMQ is reliable and correlates with objective face recognition ability. Then, using receiver operating characteristic (ROC) analyses, we attempted to determine cutoff scores on the CFMQ that would be optimal for predicting prosopagnosia, as defined by the combination of participants' age- and gender-adjusted CFMT and FFMT scores being in the lowest 2.5 th percentile. For these cutoffs, we calculated the rate (as a proportion) at which all included participants with prosopagnosia were correctly classified (i.e., sensitivity), the rate at which all those without prosopagnosia were correctly classified (i.e., specificity), and the rate at which the participants who were classified as having prosopagnosia did actually have that condition (i.e., positive predictive value), to assess the practicality of self-report for research screening purposes (see the Appendix). Next, to assess the utility of self-report in the many real-world applications for which efficient identification of individuals with below-average facial recognition abilities would be of value, we also sought to assess the sensitivity, specificity, and positive predictive value of the CFMQ and PI20 in classifying individuals as being below median (i.e., below the 50th percentile) at objective face recognition. Finally, although a diagnostic cutoff has previously been proposed for the PI20 (Shah, Gaule, et al., 2015) no comparable published reports have examined the discriminative performance of that cutoff. Therefore, we also conducted a novel analysis on data made available by Gray, Bird, and Cook (2017), to similarly determine the PI20's sensitivity, specificity, and positive predictive value for CFMT-defined developmental prosopagnosia and to compare the results with the cutoffs obtained for the CFMQ. 


\section{Method}

\section{Participant recruitment and inclusion criteria}

TestMyBrain.org hosts an online testing environment for experimental psychology. The participants in the present study were visitors to TestMyBrain.org who had freely opted to complete our experiment, among those experiments then available on the website, in exchange for feedback about their performance as compared to the performance of the prior participants. The website attracts volunteers from the general population over a wide age range, as well as of many nationalities, ethnicities, and education levels. Our study was approved by the Harvard University Institutional Review Board (protocol No. F15795-122). It conforms to the principles of the Declaration of Helsinki. In the present study, only those between the ages of 18 and 40 years old were included in the analysis, because of heteroscedasticity in the assessment scores across ages outside of, as compared to within, that age range. This heteroscedasticity was controlled by this exclusion; if this had not been done, a critical assumption of our linear regression and residualization (performed for equating scores across ages) would have been violated. This step excluded 3,376 of the original 8,242 participants. Also, only those who identified as being of European descent were included in the analyses, so as to eliminate the potential influence of the "other-race effect" (Malpass \& Kravitz, 1969; Meissner \& Brigham, 2001), given that our version of the CFMT exclusively contained face stimuli depicting faces of European descent, and prior research had suggested that the participant-stimulus ethnic match might affect CFMT scores (Bowles et al., 2009; DeGutis, Mercado, Wilmer, \& Rosenblatt, 2013). The otherrace effect is a robust phenomenon of reduced recognition ability for faces of races/ethnicities different from those of one's usual experience, so it could, theoretically, introduce spuriously low scores in our assessments for those with low experience with faces of European decent. This step excluded 1,951 of the participants remaining after the age exclusion step. Those who did not report age, gender, or ethnicity were also excluded from analysis. This step excluded 1,358 of the remaining participants. No exclusions were made on the basis of level of education, so as to allow generalization of our results to all education levels and because of prior evidence indicating that education and general intelligence are not associated with facial recognition ability (Peterson \& Miller, 2012; Wilmer, Germine, \& Nakayama, 2014). Finally, among the remaining participants, those in the top $2.5 \%$ for total time to complete our version of the CFMT were excluded, to remove participants who may have been distracted or taken a long break during this assessment, which could have degraded the maintenance in memory of the faces studied during the task. This step excluded 39 of the remaining participants.
Because the FFMT relies on long-term memory, no analogous time-based exclusion was necessary.

\section{Participants}

A total of 1,518 participants (941 female, 577 male) were included in our analyses (mean age $=27.09$ years, standard deviation $=6.44$, age range $=18-40$ ). All participants gave informed consent through the TestMyBrain.org website prior to participation.

\section{Assessments}

Three assessments of face recognition, in the following order, were included in the battery for each participant: (1) the CFMQ, (2) the CFMT, version 3 (CFMT3), and (3) an FFMT. Demographic information (age, gender, ethnicity) was also solicited from each participant.

The CFMQ is a 17-item self-report questionnaire of face recognition ability, with scores that range from 17 to 85 , where lower scores indicate poorer self-reported ability. The questions of the CFMQ were developed by some of the same researchers who developed the original version of the CFMT. Brad Duchaine, Ken Nakayama, and Laura Germine developed the CFMQ to screen for prosopagnosia, and it has been used for approximately the past decade by the Harvard Vision Lab on www.faceblind.org for this purpose. The questions were composed on the basis of their experience with persons with prosopagnosia. The CFMQ is similar to other self-report measures of facial recognition, including the better known 20-item prosopagnosia index (PI20). Similar to the PI20, each question in the CFMQ has five possible responses on a Likert scale. For the purpose of computing scores, number values of $1,2,3,4$, and 5 were associated with the five possible response options. These number values were assigned such that, on one end of the scale, the option indicating highest ability had a value of 5 , and, on the other end of the scale, the option indicating the lowest ability had a value of 1 . One's CFMQ score was, then, simply the sum of the values corresponding to one's responses across all the 17 questions. It should be noted that Questions 3, 4, 5, 6, 9, 13, 14, 15, and 17 were reverse-scored (on these questions, "always" means worse face recognition performance). The full list of questions in the CFMQ is given in the Supplementary Methods and can be compared and contrasted with the list of PI20 questions published by Shah, Gaule, Sowden, Bird, and Cook (2015a) or with the list of questions in the Kennerknecht screening questionnaire published by Kennerknecht, Plümpe, Edwards, and Raman (2007). Though the CFMQ has not served as the central focus of a publication before, questions from the CFMQ have been used in previously published studies (e.g., DeGutis, Cohan, Mercado, Wilmer, \& Nakayama, 2012; Wilmer et al., 2010) 
The CFMT3 is identical to the original version of the Cambridge Face Memory Test (CFMT) developed by Duchaine and Nakayama (2006), except that different facial stimuli are used. Instead of photographs of faces, the CFMT3 uses novel artificial faces that were generated with the FaceGen software (Singular Inversions, Toronto, ON). Since our experiment was widely and publicly available online, we refrained from using the original CFMT in our study so as to maintain the integrity of the original CFMT for clinical or inlab use - for example, as part of a diagnosis of prosopagnosia. The CFMT, version 2 (CFMT2), which also uses FaceGen computer-generated faces similar to those in our CFMT3, has been in use on faceblind.org as a screening tool for several years. For this reason, we refrained from using the CFMT2, since it is theoretically possible that our participants could have been exposed to it before. The present study is the first one published to use the CFMT3. However, another study (Wilmer et al., 2010) using a similar FaceGen-produced artificial facial stimuli CFMT variant showed a strong correlation with the original CFMT $[r(40)=$ .76, 95\% CI: .59-.86]. This correlation was not statistically different $(p=.44$, two-tailed) from the test-retest reliability $[r(387)=.70,95 \% \mathrm{CI}: .64-.74]$ reported for the original CFMT. Thus, it is likely that CFMT3 performance has a similarly high correlation with performance on the original CFMT. Additionally, in our data set, raw CFMT3 scores and raw FFMT scores (see below) correlated at $r(1516)=.47,95 \%$ CI: .43-.51, $p=1.24 \mathrm{e}-85$.

One of three possible versions of the FFMT was assigned to each participant. In all versions, participants were shown an image of a famous face on each trial and asked "Who is this?" If participants typed in a response, they were then shown the correct answer along with their response and were asked to indicate whether they had correctly identified the person. By design, this allowed for misspellings of the correct answer, or even for unique descriptions of the person, such as "Rolling Stones Singer" for "Mick Jagger," to be scored as correct. As had been done in a prior study (Wilmer et al., 2012), the total score was computed as the number of faces out of the total number of trials for which participants both (a) submitted a response and (b) verified that their response was a correct identification. Participants who did not respond with the correct name were additionally asked to indicate whether they were familiar with the person, though this does not figure into the scoring procedure. Scoring was performed automatically. We recorded all participant responses, though, so manual validation on a subsample of responses was done to verify that no systematic issues were present. To normalize the scores across FFMT versions, we calculated the FFMT version-specific $z$ score for each participant. Because the distributions of these scores from each of the FFMT versions were comparable, we treated the versions as equivalent in our analyses (see the Supplementary Methods for the full list of famous faces for each version). Thus, in this article, we refer to all three versions singularly as the FFMT.

\section{Analyses}

Identification of prosopagnosia We evaluated the discriminative utility of the CFMQ as a tool for predicting prosopagnosia, where participants were identified as having prosopagnosia independently on the basis of their combination of CFMT3 and FFMT scores. More specifically, to identify participants with prosopagnosia, we first regressed out age and gender effects (such as have been reported by Bowles et al., 2009, and Germine, Duchaine, \& Nakayama, 2011) from the raw CFMT3 and FFMT scores using a third-order polynomial regression model, so that all participants' data could be compared, regardless of the participants' age and gender. The resulting residualized scores were then translated to percentile scores, so that the resulting CFMT3 and FFMT scores could be averaged together to form a composite score. This manner of forming a composite score meets the criteria generally preferred by prosopagnosia researchers-namely, that the testing involve multiple well-validated assessments reflecting both familiar (famous) and unfamiliar facial recognition ability and that the resulting score be highly interpretable in terms of performance relative to peers.

To reflect a commonly regarded prevalence for prosopagnosia (i.e., $\sim 2.5 \%$ of the general population; Kennerknecht et al., 2006), the ground-truth designation of prosopagnosia for this study was then defined as having a composite score within the lowest-performing 2.5 percentiles among the composite scores of all the included participants. It should be noted that this approach does not rule out prosopagnosia caused by poor low/midlevel vision, poor general memory, or general social cognitive disorders (e.g., autism), which are typically ruled out in laboratory-based studies. Since there is no consensus on how prosopagnosia should be precisely defined and diagnosed, we make no strong claim that those we identified as having prosopagnosia for the purposes of our study would all have necessarily been identified as such by other research groups if brought into their labs. The conclusions of the present study are not contingent upon this issue, since the phenomena that we report with respect to the relationship of self-report to objective performance would still hold for any reasonable set of diagnostic criteria.

Evaluation of the CFMQ as a screen for prosopagnosia We assessed the discriminant utility of the CFMQ as a screening test for prosopagnosia by applying receiver operating characteristic (ROC) curve analyses (Fawcett, 2006; Green \& Swets, 1966; Spackman, 1989), which enabled quantification of the sensitivity, specificity, and positive predictive value associated with any given CFMQ cutoff score (see the Appendix). In the context of our study, sensitivity corresponds to the rate (as a 
proportion) that all included participants with prosopagnosia were correctly classified as having prosopagnosia using a given CFMQ cutoff score. Specificity corresponds to the rate that all those without prosopagnosia were correctly classified as such using a given cutoff. Finally, positive predictive value corresponds to the rate at which participants who were classified by the cutoffs as having prosopagnosia did actually have prosopagnosia. Positive predictive value is implicitly determined by the combination of sensitivity, specificity, and the positive class's prevalence. Given the intended use of such CFMQ cutoff scores as practical screening criteria for prosopagnosia researchers, a viable cutoff should yield acceptable levels of both sensitivity and positive predictive value. This is because low sensitivity would imply that many persons with prosopagnosia would be missed by the criterion, and a low positive predictive value would imply that those meeting the criterion would be unlikely to actually have prosopagnosia upon further testing. Therefore, we evaluated the practical utility of the resulting cutoffs primarily via their associated sensitivities and positive predictive values.

In particular, we first determined three optimal operating points (i.e., the optimal CFMQ cutoff scores) for detection of prosopagnosia, such that each operating point was optimized according to a different criterion. The first of the three operating points was optimized on sensitivity and specificity using Youden's $J$ index (Youden, 1950), such that the cost of loss of sensitivity was equal to the cost of loss of specificity (i.e., the operating point at which sensitivity rate + specificity rate -1 was maximized). The operating point resulting from this optimization would, in practice, be optimal in a context in which sensitivity was more important than positive predictive value (i.e., where greater importance was attached to not failing to include those with prosopagnosia than to being highly confident that one meeting the cutoff indeed had prosopagnosia). The second operating point was optimized in a more standard manner, such that the costs were proportional to the $2.5 \%$ base rate of prosopagnosia, and where the cost of loss of specificity, therefore, greatly outweighed the cost of loss of sensitivity. Thus, as compared to Youden's $J$ index, this optimization adjusted costs to reflect that those without prosopagnosia greatly outnumber those with prosopagnosia. In practice, this second criterion attempted a balance between sensitivity and positive predictive value, and so returned the main CFMQ cutoff of interest. The third operating point was the one simply yielding the maximum positive predictive value, regardless of the associated sensitivity and specificity. Thus, the three different criteria constituted different degrees of trade-off between sensitivity and positive predictive value. We then recorded the sensitivity, specificity, and positive predictive value at these operating points. The $95 \%$ confidence intervals for these three metrics were calculated using the Wilson score interval for binomial proportions (Wilson, 1927).
Identification of below-median face recognition performance A low base rate for a positive class, such as for the participants with prosopagnosia in our sample, is a disadvantage for achieving high classification performance (see the Discussion section). Additionally, the application of self-report measures of face recognition ability, such as the CFMQ, may potentially extend beyond screening for prosopagnosia to screening for below-average facial recognition ability levels of much higher prevalence (e.g., within common professions requiring daily facial recognition for successful performance). Thus, we additionally assessed the discriminant utility of the CFMQ as a screening test simply for below-median facial recognition performance. The procedure for this assessment was identical to that for the prosopagnosia screening, except that the prevalence of the positive class was set at $50 \%$ rather than at $2.5 \%$.

Assuring our ROC/cutoffs results were not due to issues with poor performance Finally, it is theoretically possible that a nonnegligible proportion of those in our sample who were identified as having prosopagnosia instead yielded artificially low scores on the CFMT3 and FFMT due to causes that were nonspecific or unrelated to their facial recognition abilities (e.g., a general visual or cognitive impairment, distraction, lack of motivation, etc.), thereby partially obscuring the full potential of the CFMQ as a screen for prosopagnosia. Under such circumstances, the discriminant utility of the CFMQ as a screen for performance in the bottom 2.5 th percentile should be lower than its ability as a screen for performance in the top 2.5 th percentile, since it is not possible that scores within the top 2.5 th percentile could be driven by such confounding factors. Therefore, we additionally assessed the CFMQ as a screen for those performing in the top 2.5th percentile on their CFMT3 + FFMT composite score, to determine whether or not there were any indications of poor data quality for those in our sample we identified as having prosopagnosia.

Evaluation of the PI20 as a screen for prosopagnosia Finally, to compare the discriminative performance of the resulting cutoffs for the CFMQ to that of another prominent self-report measure of facial recognition, we also similarly calculated the sensitivity, specificity, and positive predictive value for the cutoff score previously proposed for the PI20 (Shah, Gaule, et al., 2015) from data made available from a study by Gray, Bird, and Cook (2017). Because we could not compute age- and genderadjusted CFMT scores as we had for the CFMT3 and FFMT scores in our own sample, we simply made use of the raw CFMT scores in this analysis and of the given cutoff score previously proposed. 


\section{Results}

\section{The CFMQ is reliable and correlates with objective face recognition ability}

We verified that the CFMQ correlates with objective face recognition ability, as independently defined by CFMT3 and FFMT scores. In our sample, the Pearson's correlation between the CFMQ scores and percentile composite scores (CFMT3 and FFMT) was $r(1516)=.54,95 \%$ CI: .50-.57, $p=3.39 \mathrm{e}-$ 114 , indicating strong validity of the CFMQ as a measure of face recognition ability (Supplementary Fig. 1). Also, the Cronbach's alpha for the CFMQ scores was .91, indicating high internal consistency for this measure, similar to the other selfreports of face recognition (e.g., Cronbach's alpha for PI20 = .93, Shah, Gaule, et al., 2015; Cronbach's alpha for the Kennerknecht questionnaire $=.84$, Palermo et al., 2017).

Additional evidence for the strong validity of the self-report information contained in the CFMQ is that it correlated at least as well with each of the individual objective measures of facial recognition as the two objective measures correlated with each other. The Pearson correlation between CFMQ scores and age- and gender-adjusted (see the Method section) CFMT scores was $r(1516)=.44,95 \%$ CI: $.40-.48, p=1.06 \mathrm{e}-73$ (Supplementary Fig. 2), and the correlation between CFMQ scores and age- and gender-adjusted FFMT scores was $r(1516)=.52,95 \%$ CI: $.48-.56, p=2.68 \mathrm{e}-106$ (Supplementary Fig. 3). These two correlation values are impressive when considering that the correlation between the age- and gender-adjusted CFMT and FFMT scores was $r(1516)=.47,95 \%$ CI: $.43-.50, p=7.34 \mathrm{e}-83$ (Supplementary Fig. 4). This means that CFMQ scores were not significantly less correlated ( $p=.40$, two-tailed) with CFMT performance than FFMT performance was with CFMT performance. This also means that CFMQ scores were significantly more highly correlated ( $p=.048$, two-tailed) with FFMT performance than CFMT performance was with FFMT performance.

\section{ROC analyses: Screening for prosopagnosia}

No CFMQ cutoff score produced simultaneously adequate rates for sensitivity and positive predictive value (see
Table 1). Optimizing the CFMQ cutoff score by equating the costs of loss of sensitivity and of specificity (i.e., with Youden's $J$ index) returned a CFMQ cutoff score of 62 for a prosopagnosia screening criterion, and yielded acceptable sensitivity and specificity rates. Sensitivity was $89.19 \%$ (95\% CI: $75.29 \%-95.71 \%$ ), and specificity was $68.06 \%$ (95\% CI: $65.64 \%-70.39 \%$ ). However, this cutoff would be impractical, because the prosopagnosia prevalence of $2.5 \%$ meant that the positive predictive value achieved was only $6.52 \%$ (95\% CI: $4.68 \%-9.02 \%$ ). Thus, using this CFMQ cutoff score as a screening criterion would mean that the overwhelming majority (93.48\%) of those meeting this criterion would not actually have prosopagnosia.

Optimizing the cutoff such that the costs of loss of sensitivity and of specificity reflected the $2.5 \%$ base rate of prosopagnosia yielded a CFMQ cutoff score of 34 . At this cutoff, the positive predictive value was a respectable, albeit unreliable, $70 \%$ (95\% CI: 39.68\%-89.22\%), and specificity was $99.8 \%$ (95\% CI: 99.41\%-99.93\%). However, sensitivity was only $18.92 \%$ (95\% CI: $9.48 \%-34.21 \%$ ); thus, most of those with prosopagnosia would be missed with this criterion. Additionally, since a score of 34 or lower was so uncommon, one would rarely encounter someone with such a low score in practice. Only ten (i.e., $0.65 \%$ ) out of the 1,518 included participants had a CFMQ score this low or lower $(95 \%$ CI: $0.36 \%-1.21 \%$ ).

Finally, optimizing the cutoff by maximizing the positive predictive value alone also returned a cutoff of 34 , so the sensitivity, specificity, and positive predictive value for this optimization were identical to those immediately above.

Figure 1 plots facial recognition ability versus CFMQ score for all included participants. The performance-level cutoff corresponding to prosopagnosia is indicated with a horizontal dotted line. The two optimized cutoffs returned by the foregoing ROC analyses are indicated with vertical dotted lines. From this plot, one can get an intuitive sense of the sensitivity for a given CFMQ cutoff score by observing the proportion of participants with prosopagnosia (i.e., those below the horizontal line) that is captured by the given cutoff (i.e., those to the left of the given vertical line). Likewise, one can get an intuitive sense of the specificity by observing the proportion of

Table 1 ROC analyses of CFMQ scores screening for prosopagnosia

\begin{tabular}{llllllll}
\hline Optimization Type & Cutoff Score & Sensitivity & $95 \%$ CI & Specificity & $95 \%$ CI & PPV & $95 \%$ CI \\
\hline Youden's $J$ & 62 & $89.19 \%$ & $75.29 \%-95.71 \%$ & $68.06 \%$ & $65.64 \%-70.39 \%$ & $6.52 \%$ & $4.68 \%-9.02 \%$ \\
Standard & 34 & $18.92 \%$ & $9.48 \%-34.21 \%$ & $99.80 \%$ & $99.41 \%-99.93 \%$ & $70 \%$ & $39.68 \%-89.22 \%$ \\
Maximum PPV & 34 & $18.92 \%$ & $9.48 \%-34.21 \%$ & $99.80 \%$ & $99.41 \%-99.93 \%$ & $70 \%$ & $39.68 \%-89.22 \%$ \\
\hline
\end{tabular}

$\mathrm{PPV}=$ positive predictive value. Youden's $J$ returns the operating point at which sensitivity rate + specificity rate -1 is maximized. Thus, with this optimization, the cost of a loss in sensitivity rate is equal to the cost of a loss in specificity rate. The standard optimization is similar to Youden's $J$, except that costs are adjusted to reflect that those without prosopagnosia greatly outnumber those who with prosopagnosia. The cost of loss of specificity, therefore, greatly outweighs the cost of loss of sensitivity. In practice, this second criterion attempts a balance between sensitivity and positive predictive value, and so returns the main CFMQ cutoff of interest. 


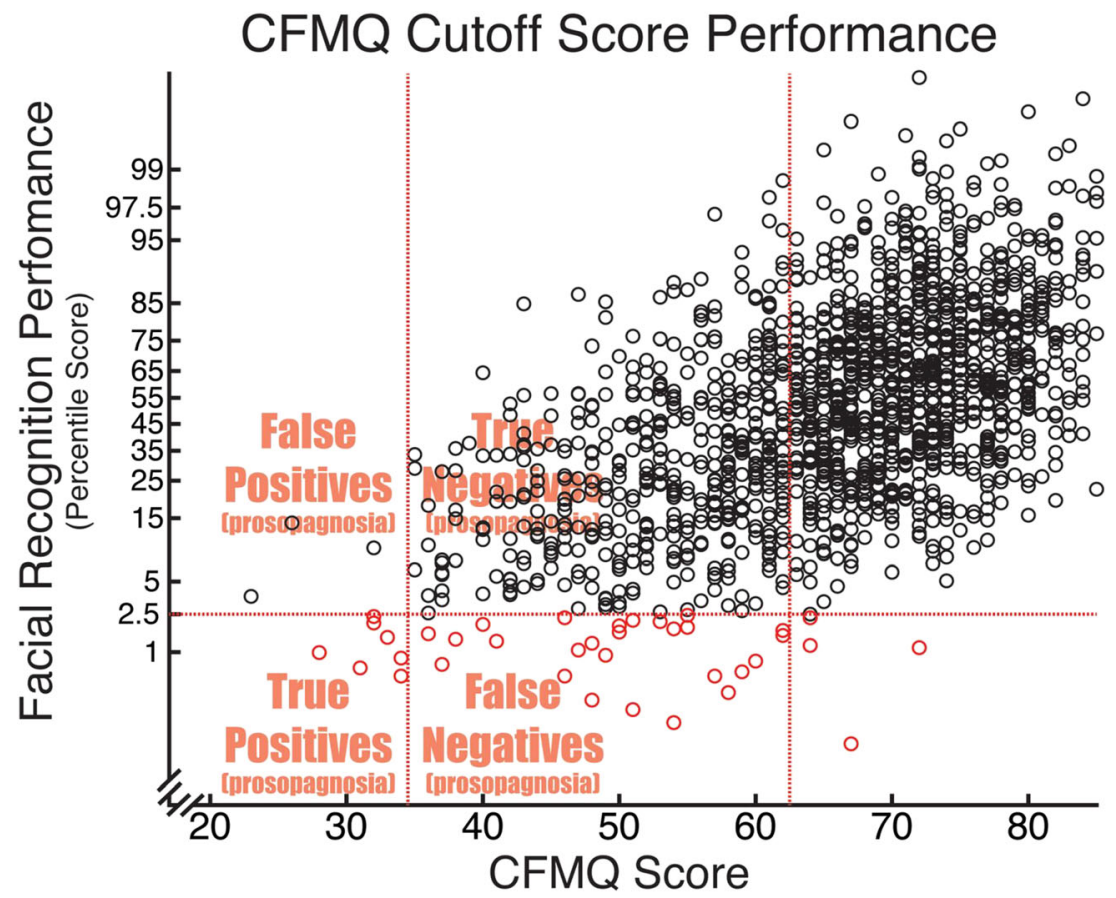

Fig. 1 Facial recognition ability versus CFMQ score. The performance level cutoff corresponding to prosopagnosia is indicated with a horizontal dotted line, such that those participants below the line were those with prosopagnosia. The two optimized CFMQ cutoffs are indicated with vertical dotted lines, such that those participants to the left of each given line were those obtaining to the given cutoff. The $x$-axis is in a $z$ transformed space to allow for ease of viewing the participants with prosopagnosia above or below the indicated cutoff scores. The sensitivity, specificity, positive predictive value, and CFMQ score prevalence rates already described can be intuited from this graph.

participants without prosopagnosia (i.e., those above the horizontal line) who surpass the CFMQ cutoff score (i.e., who fall to the right of the given vertical line). Finally, the positive predictive value corresponds to the proportion of those below the given CFMQ cutoff score (i.e., of those falling to the left of the given vertical line) who actually have prosopagnosia (i.e., who fall below the horizontal line).

\section{ROC analyses: Screening for the bottom 50th percentile}

As compared to the cutoffs optimized to screen for prosopagnosics (i.e., for performers in the bottom 2.5 th percentile), a more adequate combination of sensitivity and positive predictive value was possible for a cutoff optimized to screen for performers in the bottom 50th percentile (see Table 2). This provides evidence that the low base rate of participants with prosopagnosia was a critical disadvantage for achieving high classification performance with the CFMQ. Optimizing the cutoff such that the costs of loss of sensitivity and of specificity reflected the $50 \%$ base rate of scores within the bottom 50th percentile was equivalent to optimizing it by equating the costs of loss of sensitivity and
Sensitivity for a given CFMQ cutoff score is the proportion of participants with prosopagnosia (i.e., of those below the horizontal line) who are captured by the given cutoff (i.e., those to the left of the given vertical line). Specificity is the proportion of participants without prosopagnosia (i.e., those above the horizontal line) who surpass the CFMQ cutoff score (i.e., who fall to the right of the given vertical line). Positive predictive value is the proportion of those below the given CFMQ cutoff score (i.e., those falling to the left of the given vertical line) who actually have prosopagnosia (i.e., who fall below the horizontal line).

specificity. In this case, the optimization yielded a CFMQ cutoff score of 66 , a sensitivity of $65.35 \%$ (95\% CI: $61.89 \%-68.65 \%)$, a specificity of $71.41 \%$ (95\% CI: $68.09 \%-74.51 \%$ ), and a positive predictive value of $69.57 \%$ (95\% CI: $66.09 \%-72.83 \%$ ). The cutoff score was thus squarely above chance (i.e., 50\%) in predicting whether someone was below the 50th percentile in facial recognition performance, though depending on the particular application, the positive predictive value of approximately $70 \%$ might or might not be sufficiently predictive for the purposes of a given practical application. Regardless, that either the sensitivity or the positive predictive value was lower in the two corresponding optimizations for predicting prosopagnosia indicates that the low base rate of prosopagnosia did, in practice, disadvantage the CFMQ as a screening tool for that condition.

Optimizing the cutoff by maximizing the positive predictive value alone yielded a cutoff score of 39 (the maximum cutoff score with a positive predictive value of $100 \%$ ), a perfect specificity of $100 \%$ (95\% CI: $99.5 \%-100 \%)$, a perfect positive predictive value of $100 \%$ (95\% CI: $89.57 \%-100 \%$ ), but a sensitivity of only $4.35 \%$ (95\% CI: $3.11 \%-6.04 \%)$. That the overwhelming majority of the sensitivity was lost in order to achieve maximal specificity was reflective of the fact that 
Table 2 ROC analyses of CFMQ scores screening for the bottom 50th percentile of facial recognition ability

\begin{tabular}{llllllll}
\hline Optimization Type & Cutoff Score & Sensitivity & $95 \%$ CI & Specificity & $95 \%$ CI & PPV & $95 \%$ CI \\
\hline Youden's $J$ & 66 & $65.35 \%$ & $61.89 \%-68.65 \%$ & $71.41 \%$ & $68.09 \%-74.51 \%$ & $69.57 \%$ & $66.09 \%-72.83 \%$ \\
Standard & 66 & $65.35 \%$ & $61.89 \%-68.65 \%$ & $71.41 \%$ & $68.09 \%-74.51 \%$ & $69.57 \%$ & $66.09 \%-72.83 \%$ \\
Maximum PPV & 39 & $4.35 \%$ & $3.11 \%-6.04 \%$ & $100 \%$ & $99.5 \%-100 \%$ & $100 \%$ & $89.57 \%-100 \%$ \\
\hline
\end{tabular}

PPV = positive predictive value. Youden's $J$ returns the operating point at which sensitivity rate + specificity rate -1 is maximized. Thus, with this optimization, the cost of a loss in sensitivity rate is equal to the cost of a loss in specificity rate. The standard optimization is similar to Youden's $J$, except that costs are adjusted to reflect that those without prosopagnosia greatly outnumber those who with prosopagnosia. The cost of loss of specificity, therefore, greatly outweighs the cost of loss of sensitivity. In practice, this second criterion attempts a balance between sensitivity and positive predictive value, and so returns the main CFMQ cutoff of interest.

the distribution of CFMT3 scores for those in the bottom 50 percentiles greatly overlaps that for those in the top 50 percentiles (compare those above and below the 50th percentile in facial recognition ability in the scatter plot in Fig. 1).

\section{ROC analyses: Screening for the top 2.5th percentile}

The discriminant utility of the cutoff scores optimized to screen for performers in the top 2.5th percentile of the objective assessment was not higher than those optimized for screening for prosopagnosia (see Table 3). This means that there was no indication of poor data quality for those in our sample we identified as having prosopagnosia, so poor data quality could not explain the low discriminant utility of the relevant cutoff scores for prosopagnosia. Optimizing the CFMQ cutoff score using Youden's $J$ index returns a CFMQ cutoff score of 72 for a top 2.5th percentile performance screening criterion, yielding a sensitivity at $76.92 \%(95 \%$ CI: $61.66 \%-87.35 \%)$ and a specificity at $67.68 \%$ (95\% CI: $65.25 \%-70.02 \%$ ). However, this criterion was impractical because, as was the case with the screening of prosopagnosia under this cutoff optimization method, the prevalence of our top performers at $2.5 \%$ meant that the positive predictive value achieved here was similarly only $5.91 \%$ (95\% CI: $4.17 \%-$ $8.31 \%)$. The sensitivity, specificity, and positive predictive value for this optimization are comparable with, if somewhat numerically lower than, the corresponding optimization for prosopagnosia screening. Thus, using this CFMQ cutoff score as a screening criterion would likewise mean that the overwhelming majority $(94.09 \%)$ of those meeting this criterion would not actually perform within the top 2.5 th percentile.

Optimizing the cutoff such that the costs of loss of sensitivity and of specificity reflected the $2.5 \%$ base rate of the top performers yielded a CFMQ cutoff at the empirical maximum score of 85 . At this cutoff, the positive predictive value was $42.86 \%$ (95\% CI: $15.82 \%-74.95 \%)$, and the specificity was $99.73 \%$ (95\% CI: $99.31 \%-99.89 \%)$. However, sensitivity was only $7.69 \%$ (95\% CI: $2.65 \%-20.32 \%$ ); thus, most of the top performers were missed with this criterion. The sensitivity, specificity, and positive predictive value for this optimization are again comparable with, though numerically lower than, the corresponding optimization for prosopagnosia screening. Additionally, since a score of 85 is likewise very uncommon, one would rarely encounter someone with such a high score. Only seven (i.e., $0.46 \%$ ) out of the 1,518 included participants had this CFMQ score. Finally, optimizing the cutoff by maximizing the positive predictive value alone also returned a cutoff of 85 , so the sensitivity, specificity, and positive predictive value for this optimization were identical to those reported immediately above.

Low performance scores on the CFMT3 and FFMT could, theoretically, be reflective of poor data quality, though the same is not true of high performance scores. Therefore, a stronger discriminant utility of CFMQ scores for detecting our highest than for our lowest "prosopagnosia" performers, if it existed, could have been an indication of compromised data quality for our lowest performers. However, since the

Table 3 ROC analyses of CFMQ scores screening for the top 2.5th percentile of facial recognition ability

\begin{tabular}{llllllll}
\hline Optimization Type & Cutoff Score & Sensitivity & $95 \%$ CI & Specificity & $95 \%$ CI & PPV & $95 \%$ CI \\
\hline Youden's $J$ & 72 & $76.92 \%$ & $61.66 \%-87.35 \%$ & $67.68 \%$ & $65.25 \%-70.02 \%$ & $5.91 \%$ & $4.17 \%-8.31 \%$ \\
Standard & 85 & $7.69 \%$ & $2.65 \%-20.32 \%$ & $99.73 \%$ & $99.31 \%-99.89 \%$ & $42.86 \%$ & $15.82 \%-74.95 \%$ \\
Maximum PPV & 85 & $7.69 \%$ & $2.65 \%-20.32 \%$ & $99.73 \%$ & $99.31 \%-99.89 \%$ & $42.86 \%$ & $15.82 \%-74.95 \%$
\end{tabular}

$\mathrm{PPV}=$ positive predictive value. Youden's $J$ returns the operating point at which sensitivity rate + specificity rate -1 is maximized. Thus, with this optimization, the cost of a loss in sensitivity rate is equal to the cost of a loss in specificity rate. The standard optimization is similar to Youden's $J$, except that costs are adjusted to reflect that those without prosopagnosia greatly outnumber those who with prosopagnosia. The cost of loss of specificity, therefore, greatly outweighs the cost of loss of sensitivity. In practice, this second criterion attempts a balance between sensitivity and positive predictive value, and so returns the main CFMQ cutoff of interest. 
sensitivity, specificity, and positive predictive value metrics yielded by the optimizations of the screening for the top performers were comparable to, and numerically even lower than, those corresponding to the optimizations screening for prosopagnosia, we thus found no evidence that the low metrics in the prosopagnosia optimizations were due to poor data quality for the subsample of our participants who were identified as having prosopagnosia. For this reason, we can be confident that those we identified as having prosopagnosia do indeed have face recognition impairment and are representative of the population of those with prosopagnosia.

\section{Item response theory analyses}

It is possible that the low discriminant utility of the CFMQ we observed at one or both of its high and/or low ends could have been due to lack of precision for reliably identifying exceptionally high or low self-assessed face recognition. For example, perhaps the CFMQ, which was originally developed to identify poor face recognition, is more sensitive at its low than at its high end. Another possibility is that the CFMQ's high correlation with objective measures results from a high capacity to discriminate self-assessed face recognition within the normal range of performance, but this discrimination capacity breaks down at the extremes; another way of stating this is that the CFMQ might be better at discriminating the 49th from the 50th percentile of self-assessed face recognition than at discriminating the 2 nd from the 3 rd percentile, or the 98th from the 97 th percentile. Finally, it was worth checking to verify that the overall precision of the CFMQ, across its full range of values, but especially at its extremes, was reasonably comparable to that of our objective measures.

To address these three questions - (1) a potential differential precision of the CFMQ at the high versus the low end, (2) the breakdown of precision for the CFMQ at its extremes, and (3) potentially poor precision of the CFMQ relative to the objective measures - we conducted a standard item-response theory analysis that would enable a direct quantification of the precision of the instrument across its full range (Wilmer et al., 2012). The details of these results are included as supplemental information. The conclusions of these analyses were that (1) the CFMQ is similarly precise at both the high and low ends; (2) the CFMQ is actually more, rather than less, sensitive at its extremes than in its middle range; and (3) the CFMQ has comparable, or even better, precision relative to our objective measures across its full range, including at the extremes. Therefore, we concluded that the CFMQ has good precision across its whole range, that the high-end analysis was not limited by poor CFMQ precision in this range, and that the limited diagnostic and screening utility we observed for self-report does not appear to be a simple artifact of the limited precision of self-report instruments.
Our item-response theory analysis also confirmed (see the supplemental information) that the sensitivities of both the CFMT3 and FFMT are similar at both extremes of performance, thereby effectively ruling out the possibility that these instruments, which were developed in part to identify poor face recognition performance, are simply insensitive to high performance, thereby limiting the comparative value of our high-end ROC analysis.

\section{Single question screening}

The first question in the CFMQ is "Compared to my peers, I think my face recognition skills are . . ," with possible responses from $1=$ far below average to $5=$ far above average, which constitutes the most general and direct self-assessment item for face recognition ability in the questionnaire. Use of the "far below average" response on this item as a prosopagnosia screening criterion yielded, arguably, the most acceptable combination of sensitivity, positive predictive value, and prevalence yet. The sensitivity was $54.05 \%$ (95\% CI: $41.27 \%-66.32 \%$ ), the specificity was $92.1 \%$ (95\% CI: $88.89 \%-94.44 \%$ ), the positive predictive value was $14.6 \%$ (95\% CI: $9.65 \%-21.48 \%$ ), and the cutoff contained $9.03 \%$ (95\% CI: $7.69 \%-10.57 \%)$ of our included participants. Therefore, use of this single response might be relatively more practical than use of the sum score on the entire CFMQ as a screening criterion for prosopagnosia. Nonetheless, this criterion still might not be highly useful in absolute terms, since the positive predictive value suggests that most persons meeting this criterion $(85.4 \%)$ would not have prosopagnosia upon further objective performance testing.

\section{PI20 cutoff analysis on Gray, Bird, and Cook (2017)}

The discriminant utility of our CFMQ cutoff scores can be compared with the discriminant utility of the PI20 cutoff score proposed by the authors of the PI20. Combining both of the data sets reported in Gray, Bird, and Cook (2017), we conducted a novel analysis of the discriminant utility of the PI20 cutoff score of 65 or greater as a screen for prosopagnosia. Our calculations yielded a total of 425 included participants, 59 of whom we identified as having prosopagnosia on the basis that they obtained a score of 44 or lower on the original version of the CFMT, which, from previously published norms (Bowles et al., 2009; Duchaine \& Nakayama, 2006; Duchaine, Yovel, Butterworth, \& Nakayama, 2006; Duchaine, Yovel, \& Nakayama, 2007; Garrido, Duchaine, \& Nakayama, 2008), corresponds to performance approximately within the bottom 2.5\% (no FFMT was administered). A total of 12 participants had been classified as having prosopagnosia with the PI20 cutoff, five of whom actually had prosopagnosia according to their CFMT score. Thus, the positive predictive value was $5 / 12=41.67 \%$, though the small number of participants 
classified as having prosopagnosia with the PI20 cutoff meant that the confidence interval on this rate was wide $(95 \% \mathrm{CI}$ : $19.33 \%-68.05 \%$ ). The sensitivity was $5 / 59=8.47 \%$ (95\% CI: $3.67 \%-18.35 \%$ ), and the specificity was $359 / 366=98.09 \%$ (95\% CI: $96.11 \%-99.07 \%$ ). Given that 12 out of the 425 participants met the PI20 cutoff, the prevalence of those with such scores was thus $2.82 \%$ (95\% CI: $1.62 \%-4.87 \%)$. Given the wide confidence interval on the positive predictive value, it was not possible to adequately evaluate the usefulness of the PI20 cutoff for predicting CFMT-defined prosopagnosia. However, it is clear that the sensitivity was low, indicating that most (about $91.5 \%$ ) of those with prosopagnosia would not be detected.

The PI20 cutoff within the Gray, Bird, and Cook (2017) data set thus yielded sensitivity, specificity, and positive predictive values comparable to those reported above for the CMFQ cutoff of 34 within our data set (see Table 4). Though sensitivity, specificity, and positive predictive value were all numerically lower for the PI20 cutoff than for that CMFQ cutoff, the confidence intervals for these metrics greatly overlapped, indicating that they did not differ significantly between the two questionnaires. This holds true even if we identify our participants with prosopagnosia only on the basis of CFMT3 scores instead of on the basis of composite CFMT3 and FFMT scores (Gray et al. did not administer the FFMT to their participants). Specifically, performing the analogous cutoff optimization for CFMT3-defined prosopagnosia as for composite-score-defined prosopagnosia again yielded a CFMQ cutoff score of 34, with a sensitivity of $16.22 \%(95 \%$ CI: $7.65 \%-31.14 \%$ ), specificity of $99.73 \%$ (95\% CI: $99.31 \%-$ $99.89 \%$ ), and positive predictive value of $60 \%$ (95\% CI: $31.27 \%-83.18 \%$ ). The one advantage that the PI20 cutoff has over the CFMQ cutoff, though, is that the prevalence of those meeting the cutoff was higher $(2.82 \%$ vs. $0.66 \%$, respectively), and the difference was statistically significant according to a binomial test on the Gray, Bird, and Cook (2017) data, for which the null hypothesis was that the prevalence was equal to our value of $0.66 \%(p=.0000070)$.

Figure 2 plots the data of Gray, Bird, and Cook (2017) in a manner analogous to our Fig. 1. One difference to note in Fig. 2 as compared to Fig. 1 is that those meeting the PI20 cutoff are those to the right of the vertical line, not to the left, as was the case for those meeting the CFMQ cutoff in Fig. 1. Also, the $y$-axis is the CFMT score, not the composite (CFMT3 + FFMT) percentile score we were able to calculate in our own data. Finally, the $y$-axis space did not need to be transformed, since CFMT scores were already normally distributed, allowing for easy visibility of the participants with CFMTdefined prosopagnosia who fell above and below the proposed PI20 cutoff score.

\section{Discussion}

We investigated the utility of a self-report measure of facial recognition ability, the Cambridge Face Memory Questionnaire (CFMQ), as a screen for discriminating between individuals with and without prosopagnosia-level performance on objective tests commonly used to confirm a diagnosis of developmental prosopagnosia. We found the CFMQ to be a highly reliable and valid index of facial recognition ability [Cronbach's alpha $=.91$ and Pearson's $r(1516)=$ .54 with objective performance]. The validity of the CFMQ as a measure of facial recognition is impressive given that the reported average correlation between self-evaluated memory capability and objective recognition performance metrics in other domains of memory is only $r=.1$ (Beaudoin \& Desrichard, 2011; Wilmer, 2017). Nonetheless, no CFMQ cutoff score yielded a practically adequate combination of sensitivity and positive predictive value for prosopagnosia, as independently determined by performance on the objective facial recognition tests. In other words, no CFMQ cutoff score both (1) included most of the participants with prosopagnosia and (2) produced a high and reliable rate at which those participants who met the cutoff actually had prosopagnosia. That higher simultaneous rates for sensitivity and positive predictive value were possible with a CFMQ cutoff score optimized for discriminating between those above and below median performance on the objective tests indicates that the low base rate of prosopagnosia is a source of the poor discrimination between those with and without prosopagnosia. Furthermore, that comparable rates for sensitivity and positive predictive value were produced with a CFMQ cutoff score optimized for discriminating the top 2.5 percentile on the objective tests from the rest of the participants means that there was no indication that the quality of the data for those we had identified as having prosopagnosia was poor. Finally, that comparable discriminative utility was found also for the cutoff score proposed for the PI20 suggests that the low discriminative utility of the CFMQ for prosopagnosia generalizes to other selfreport measures.

Table 4 ROC analyses of PI20 scores screening for CFMT-defined prosopagnosia

\begin{tabular}{lllllll}
\hline Cutoff Score & Sensitivity & $95 \%$ CI & Specificity & $95 \%$ CI & PPV & $95 \%$ CI \\
\hline 65 & $8.47 \%$ & $3.67 \%-18.35 \%$ & $98.09 \%$ & $96.11 \%-99.07 \%$ & $41.67 \%$ & $19.33 \%-68.05 \%$ \\
\hline
\end{tabular}

$\mathrm{PPV}=$ positive predictive value 


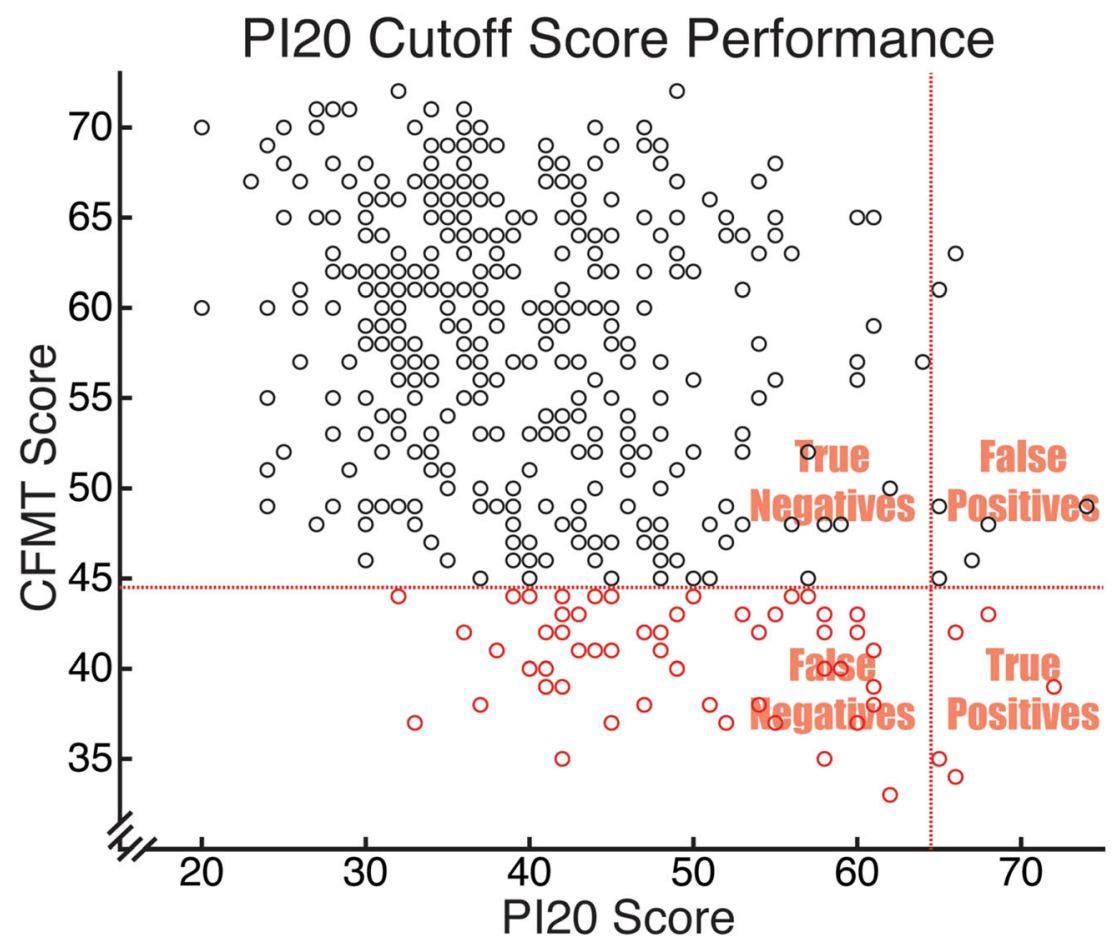

Fig. 2 CFMT score versus PI20 score. The CFMT score corresponding to prosopagnosia is indicated with a horizontal dotted line, such that those participants below the line were those with CFMT-defined prosopagnosia. The proposed PI20 cutoff is indicated with a vertical dotted line, such that those participants to the right of the line were those obtaining the cutoff. The sensitivity, specificity, positive predictive value, and CFMQ score prevalence rates already described can be intuited from this graph. Sensitivity for the PI20 cutoff score is the proportion of participants with CFMT-defined prosopagnosia (i.e., of those below the

The utility of any given screening cutoff score may depend on the context of the research and the preferences of the researcher. For example, a researcher may be seeking to maximize their sample of individuals with prosopagnosia and may have the resources to perform confirmatory diagnostic tests on many individuals even if it is expected that many of those individuals will not ultimately be found to have prosopagnosia. Such a researcher would likely value sensitivity over positive predictive value with their screening cutoff score. A researcher seeking to be more confident that an individual meeting the cutoff will actually have prosopagnosia, even if it means failing to detect many of those with prosopagnosia, will likely value positive predictive value over sensitivity. For this reason, we optimized the CFMQ cutoff according to different criteria that favored either sensitivity or specificity, or aimed to strike a balance between the two. Cutoff optimization favoring sensitivity yielded a cutoff score with an associated sensitivity of $89.19 \%$, but positive predictive value of only $6.52 \%$. Cutoff optimization to strike more of a balance of sensitivity and positive predictive value yielded a cutoff with an associated sensitivity of only $18.92 \%$ and positive predictive value of $70 \%$. Though $70 \%$ is a respectable rate for a positive predictive value, it must be noted that this horizontal line) who are captured by the cutoff (i.e., those to the right of the vertical line). Specificity is the proportion of participants without prosopagnosia (i.e., those above the horizontal line) who do not obtain the PI20 cutoff score (i.e., who fall to the left of the horizontal line). Positive predictive value is the proportion of those obtaining the PI20 cutoff score (i.e., of those falling to the right of the vertical line) who actually have CFMT-defined prosopagnosia (i.e., who fall below the horizontal line).

was not a highly reliable value (95\% CI: $39.68 \%-89.22 \%$ ). Furthermore, those meeting that cutoff included only $0.65 \%$ of all participants. Cutoff optimization favoring positive predictive value yielded the same results yielded in the attempt to strike a balance between sensitivity and positive predictive value. Notwithstanding differences in the value that researchers may place on sensitivity versus positive predictive value, no CFMQ cutoff is likely to be practical. The researcher who values sensitivity can obtain good sensitivity, but will find that more than $93 \%$ of persons meeting the cutoff optimized for sensitivity will not actually have prosopagnosia. The researcher who values positive predictive value will rarely be able to find individuals meeting the cutoff optimized for positive predictive value. Finally, the researcher seeking a balance between sensitivity and positive predictive value is left without a cutoff that is able to achieve it.

That the CFMQ is not apt for discriminating between those with and without prosopagnosia may seem inconsistent with its demonstrated reliability and validity as an index of objective facial recognition ability. However, this lack of discriminative utility stems from the low base rate of prosopagnosia (here, $2.5 \%$ ), along with the fact that developmental prosopagnosia constitutes the tail end of a roughly normal 
distribution of facial recognition ability, rather than some extreme cluster largely segregated from the rest of the distribution, when diagnosed on the basis of behavior alone (Barton \& Corrow, 2016). For these reasons, an exceptionally high degree of precision in the prediction of objective performance from self-report would be required for adequate discrimination of the small number of those with prosopagnosia from the nearly 40 -fold larger number of those without prosopagnosia. Though the association between self-report and objective performance is robust, it is not commensurate with the extremely high degree of precision required, meaning that the distribution of CFMQ scores for those without prosopagnosia greatly overlaps with and drowns out the distribution of CFMQ scores for those with prosopagnosia. This state of affairs is plainly evident in Fig. 1, where, despite the clearly robust linear relationship between CFMQ score and objective performance, there is consistent overlap in CFMQ scores between those with and without prosopagnosia. An indication that the low base rate of prosopagnosia exacerbates the situation is our finding that when using the CFMQ instead merely to discriminate between the upper and lower $50 \%$ of facial recognition performers, higher simultaneous rates for sensitivity $(65.35 \%)$ and positive predictive value $(69.57 \%)$ can be achieved with an optimized cutoff score.

Our participants performed all assessments remotely over the internet, and prior evidence indicates that the quality and reliability of data collected through the web is as good as that of data collected in the laboratory (Germine et al., 2012). Consistent with this finding, the magnitude of our correlation between CFMQ scores and objective facial recognition performance fell at the high end of the range of values previously reported for associations between subjective and objective measures in the laboratory-based studies of other research groups (Bindemann et al., 2014; Gray et al., 2017; Livingston \& Shah, 2017; Rotshtein et al., 2007; Shah, Gaule, et al., 2015a; Shah, Sowden, et al., 2015b; Stollhoff et al., 2011; Turano et al., 2016; Turano \& Viggiano, 2017). Nonetheless, one could speculate that the data for the participants we identified as having prosopagnosia might be uniquely suspect with respect to its quality, given that this subset of participants, by definition, constitutes the outliers, and that such poor performance could merely be caused by factors unrelated to facial recognition ability. If this were so, the apparent utility of the CFMQ for discriminating between those with and without prosopagnosia would be spuriously reduced in our study. However, this possibility is highly unlikely, given that in using the CFMQ to attempt to discriminate the top $2.5 \%$ of facial recognition performers from the rest of our participants, we discovered comparably poor discriminative ability as we had found for prosopagnosia. Furthermore, additional item response theory analyses (Supplementary Results, Supplementary Figs. 1-5) of all of our assessment metrics verified that facial recognition ability estimate precisions from each of the metrics we employed were sufficient within the relevant score ranges. Thus, neither compromised quality of our participant sample nor of our assessment metrics were the cause for the CFMQ being poor at discriminating between those with and without prosopagnosia. However, even considering the hypothetical case in which a significant proportion of the participants that we identified as having prosopagnosia were due to more general vision, memory, or social cognitive deficits, the inclusion of such participants would still have negligible influence on the results and interpretation of our study. This is because all of these conditions still bear upon a person's ability to recognize faces relative to others in the population, and whether one's facial recognition deficit is domain-specific or not, comparable impairment should still be evident in the objective assessments and in self-report.

Does the limited discrimination of prosopagnosia-level performance observed here generalize broadly across selfreport instruments? As we previously mentioned, we found the magnitude of the correlation between the CFMQ and objective performance to be higher than almost all prior published correlations between subjective and objective measures of facial recognition. This is perhaps merely because of the steps we took to make our metric of objective performance standardized and reliable (e.g., age and gender adjustments, percentile scoring, and the composition of two tests), rather than because the CFMQ reflects objective performance more strongly than do other self-report measures. Nonetheless, for the reasons mentioned above relating to the low base rate of prosopagnosia and the overlap in self-report scores between those with and without prosopagnosia, it seems unlikely that any of the other self-report measures currently in existence would be capable of achieving substantially better discriminative ability than the CFMQ. Consistent with this is our finding from a novel analysis of data from another self-report measure, the PI20 (Shah, Gaule, et al., 2015b). Using data available from a published study (Gray et al., 2017) in which PI20 and CFMT scores were collected from a sample of 435 participants, we found that the proposed PI20 score cutoff of 65 yielded rates of sensitivity, specificity, and positive predictive value comparable to, if numerically lower than, those yielded by the cutoff score of 34 for the CFMQ, even when prosopagnosia was analogously defined only on the basis of CFMT3 scores, rather than composite scores, within our participant sample. In addition to suggesting the generalizability of our findings to other self-report measures, this finding further argues against the possibility that our data set may in some way be contaminated by nonconscientious participants, since, unlike our internet-based participant sample, the Gray, Bird, and Cook's participant sample were laboratory-based, and so close supervision was possible during testing.

The poor performance of the CFMT and the PI20 in discriminating between those with and without prosopagnosia 
can be contrasted with the much better performance, for example, of the autism-spectrum quotient (AQ; Baron-Cohen, Wheelwright, Skinner, Martin, \& Clubley, 2001) in discriminating between those with and without autism spectrum disorders, or of the Montreal Cognitive Assessment (MoCA) in discriminating between those with and without mild cognitive impairment or Alzheimer's disease. The AQ has been reported to detect $80 \%$ of participants with high-functioning autism spectrum disorders when a cutoff score of 32 or higher on the AQ was used, whereas only $2 \%$ of controls had such scores (i.e., a cutoff sensitivity of $80 \%$ and specificity of 98\%; Baron-Cohen et al., 2001). Scores on the Montreal Cognitive Assessment (MoCA) have been reported to enable a detection rate (i.e., sensitivity) of $81 \%$, a correct rejection rate (i.e., specificity) of $77 \%$, and positive prediction at a rate of $78 \%$ for mild cognitive impairment. MoCA scores also enable a sensitivity of $88 \%$, specificity of $98 \%$, and positive predictive value of $98 \%$ for Alzheimer's disease (Freitas, Simões, Alves, \& Santana, 2013).

Comparisons with the AQ and the MoCA may not be appropriate, however. Critical differences between how autism spectrum disorders and dementias are screened and diagnosed as compared to how prosopagnosia tends to be screened and diagnosed likely explain why the AQ and MoCA are not susceptible to the same disadvantages that self-reported facial recognition has for predicting prosopagnosia. Autism spectrum disorders are diagnosed on the basis of semistructured observation of behavior by an examiner and the questions in the AQ are similar in content to this diagnostic assessment. Prosopagnosia, on the other hand, tends to be diagnosed not on the basis of ecologically observed behaviors corresponding to those subjectively assessed with the CFMT or PI20, but rather on the basis of objective performance metrics of facial recognition, (e.g., the CFMT and FFMT; though see Kennerknecht et al., 2006; Kennerknecht, Nga, \& Wong, 2008a; Kennerknecht, Pluempe, \& Welling, 2008b; Stollhoff et al., 2011). Alzheimer's disease and other dementias, like prosopagnosia, are diagnosed on the basis of objective tests; however, the MoCA is itself an objective measure, not a subjective self-report measure, and so, like the $A Q$, is similar in nature to the relevant diagnostic tests. Thus, the AQ and MoCA are not susceptible to the reduction of discriminative performance that is likely inevitably introduced when there is a difference in the kind of information (i.e., subjective/observational vs. objective performance) used between screening and diagnosis.

If all forms of self-report, by themselves, are of limited value in screening for prosopagnosia, then what alternative screening approaches could be practicable for researchers seeking to identify and recruit persons with prosopagnosia? Our study may serve as a proof of concept for an approach of remote objective performance testing via the internet. Though the current gold-standard assessments of facial recognition performance (e.g., the CFMT original and FFMT) may be reserved for laboratory-based confirmation of prosopagnosia, collecting data from alternative forms of such objective performance assessments online and then screening based on performance scores could serve as an efficient, economical, and reliable means of locating persons highly likely to have prosopagnosia. Indeed, alternative forms of the CFMT, along with norms for performance within the general population, already exist that may be suitable for such purposes (e.g., the CFMT3 and the norms from the present study, or the CFMT2 and the norms from Germine, Duchaine, \& Nakayama, 2011, 2013).

Though self-report information, by itself, is of limited use for predicting prosopagnosia as independently defined by objective tests, this does not preclude it as an integral aspect of the very definition of prosopagnosia, and thus, as being essential in the context of diagnosis. Because self-report often reflects aspects of everyday functioning and disability not fully captured by objective tests (Duchaine \& Weidenfeld, 2003; Stollhoff et al., 2011), self-report could potentially be regarded either as the primary diagnostic information or, at least, as on par with objective measures. This possibility of using selfreport as primary diagnostic information for prosopagnosia is similar to how self-report, in the form of extended structured clinical interviews, is the primary or co-primary diagnostic information for disorders of which subjective distress and/or functional disability are, by definition, essential characteristics. Developmental disorders such as autism, dyslexia, and attention deficit hyperactivity disorder, as well as psychiatric disorders such as depression, obsessive-compulsive disorder, and posttraumatic stress disorder, are examples of such disorders. Therefore, some prosopagnosia researchers treat subjective report as the primary information for a putative diagnosis (e.g., Kennerknecht et al., 2006; Kennerknecht, Pluempe, et al., 2008a; Kennerknecht et al., 2007; Stollhoff et al., 2011), though it should be noted that prosopagnosia researchers using this approach are currently in the minority.

Alternatively, self-report could instead be used as a prerequisite for the objective tests of facial recognition that are then subsequently used as the primary information to confirm the diagnosis of prosopagnosia. Such a step-wise approach has precedent in screening for cognitive decline, for which objective measures are collected only if self- or informant-reported complaints exist (Crooks, Buckwalter, Petitti, Brody, \& Yep, 2005; Winblad et al., 2004). In line with this approach, most labs studying prosopagnosia have employed some form of self-report or self-identification as a screen to select individuals for further laboratory testing, where objective measures are then collected as the ground-truth information to perform the putative diagnosis. 
Presently, no consensus exists within the research community for how self-report information should be utilized in the diagnosis of prosopagnosia. However, our data reveal that this unresolved detail is not without significant consequences. For the purposes of our study, we identified persons with prosopagnosia within our sample entirely on the basis of their performance on objective tests typically utilized in the confirmation of a diagnosis of prosopagnosia. It is evident that many of the participants that we identified as having prosopagnosia instead subjectively perceived their facial recognition ability to be within the normal, or even high, range, as compared to others (see Fig. 1). To meet a "true" diagnosis, should those even without insight into their exceptionally poor facial recognition ability be considered as having prosopagnosia, or should some form of subjective complaint instead be required? Likewise, for those whose performance on objective measures is within the normal range, but who nonetheless present with complaints that their ability is exceptionally impaired, with real-world consequences, how much, if any, provision should be made for diagnosing such individuals with prosopagnosia? The data from the present study cannot resolve this issue of diagnostic convention, but they do highlight some of what is at stake in any convention that might be adopted.

Acknowledgements Research reported in this publication was supported by the National Eye Institute of the National Institutes of Health under award number R01EY026057

\section{Appendix: ROC analysis definitions in the context of detecting prosopagnosia with the CFMQ}

Sensitivity The rate at which participants with prosopagnosia were correctly classified when using a given CFMQ cutoff score. A low sensitivity rate would imply that a high proportion of persons with prosopagnosia would not be detected on the basis of their CFMQ score alone.

Specificity The rate at which participants without prosopagnosia were correctly classified when using a given CFMQ cutoff score. A low specificity rate would imply that a high proportion of persons without prosopagnosia would be falsely labeled as having prosopagnosia on the basis of their CFMQ score alone.

Positive predictive value The rate at which those classified as having prosopagnosia actually had prosopagnosia. High positive predictive value does not imply high sensitivity, or vice versa, so it is possible for a high proportion of persons with prosopagnosia to fail to be detected even if a high proportion of those classified as having prosopagnosia actually have prosopagnosia.

Publisher's note Springer Nature remains neutral with regard to jurisdictional claims in published maps and institutional affiliations.

\section{References}

Baron-Cohen, S., Wheelwright, S., Skinner, R., Martin, J., \& Clubley, E. (2001). The Autism-Spectrum Quotient (AQ): Evidence from Asperger syndrome/high-functioning autism, males and females, scientists and mathematicians. Journal of Autism and Developmental Disorders, 31, 5-17. doi:https://doi.org/10.1023/A: 1005653411471

Barton, J. J. S., \& Corrow, S. L. (2016). The problem of being bad at faces. Neuropsychologia, 89, 119-124. doi:https://doi.org/10.1016/ j.neuropsychologia.2016.06.008

Beaudoin, M., \& Desrichard, O. (2011). Are memory self-efficacy and memory performance related? A meta-analysis. Psychological Bulletin, 137, 211-241. doi:https://doi.org/10.1037/a0022106

Bindemann, M., Attard, J., \& Johnston, R. A. (2014). Perceived ability and actual recognition accuracy for unfamiliar and famous faces. Cogent Psychology, 1, 986903. doi:https://doi.org/10.1080/ 23311908.2014.986903

Bobak, A. K., Mileva, V. R., \& Hancock, P. J. B. (2018). Facing the facts: Naive participants have only moderate insight into their face recognition and face perception abilities. Quarterly Journal of Experimental Psychology. Advance online publication. doi:https:// doi.org/10.1177/1747021818776145

Bowles, D. C., McKone, E., Dawel, A., Duchaine, B., Palermo, R., Schmalzl, L., . . Y Yovel, G. (2009). Diagnosing prosopagnosia: Effects of ageing, sex, and participant-stimulus ethnic match on the cambridge face memory test and cambridge face perception test. Cognitive Neuropsychology, 26, 423-455. doi:https://doi.org/10. 1080/02643290903343149

Crooks, V. C., Buckwalter, J. G., Petitti, D. B., Brody, K. K., \& Yep, R. L. (2005). Self-reported severe memory problems as a screen for cognitive impairment and dementia. Dementia, 4, 539-551. doi:https:// doi.org/10.1177/1471301205058310

De Heering, A., \& Maurer, D. (2014). Face memory deficits in patients deprived of early visual input by bilateral congenital cataracts. Developmental Psychobiology, 56, 96-108. doi:https://doi.org/10. 1002/dev.21094

DeGutis, J., Cohan, S., Mercado, R. J., Wilmer, J., \& Nakayama, K. (2012). Holistic processing of the mouth but not the eyes in developmental prosopagnosia. Cognitive Neuropsychology, 29, 419-446. doi:https://doi.org/10.1080/02643294.2012.754745

DeGutis, J., Mercado, R. J., Wilmer, J., \& Rosenblatt, A. (2013). Individual differences in holistic processing predict the own-race advantage in recognition memory. PLoS ONE, 8, e58253. doi: https://doi.org/10.1371/journal.pone.0058253

Duchaine, B. C., \& Weidenfeld, A. (2003). An evaluation of two commonly used tests of unfamiliar face recognition. Neuropsychologia, 41, 713-720. doi:https://doi.org/10.1016/S0028-3932(02)00222-1

Duchaine, B. C., Yovel, G., Butterworth, E. J., \& Nakayama, K. (2006). Prosopagnosia as an impairment to face-specific mechanisms: Elimination of the alternative hypotheses in a developmental case. Cognitive Neuropsychology, 23, 714-747. doi:https://doi.org/10. 1080/02643290500441296

Duchaine, B., \& Nakayama, K. (2006). The Cambridge Face Memory Test: Results for neurologically intact individuals and an investigation of its validity using inverted face stimuli and prosopagnosic participants. Neuropsychologia, 44, 576-585. doi:https://doi.org/ 10.1016/j.neuropsychologia.2005.07.001

Duchaine, B., Yovel, G., \& Nakayama, K. (2007). No global processing deficit in the Navon task in 14 developmental prosopagnosics. Social Cognitive and Affective Neuroscience, 2, 104-113. doi: https://doi.org/10.1093/scan/nsm003

Fawcett, T. (2006). An introduction to ROC analysis. Pattern Recognition Letters, 27, 861-874. doi:https://doi.org/10.1016/j. patrec.2005.10.010 
Freitas, S., Simões, M. R., Alves, L., \& Santana, I. (2013). Montreal cognitive assessment: Validation study for mild cognitive impairment and alzheimer disease. Alzheimer Disease and Associated Disorders, 27, 37-43. doi:https://doi.org/10.1097/WAD. 0b013e3182420bfe

Garrido, L., Duchaine, B., \& Nakayama, K. (2008). Face detection in normal and prosopagnosic individuals. Journal of Neuropsychology, 2, 119-140. doi:https://doi.org/10.1348/ $174866407 X 246843$

Germine, L. T., Duchaine, B., \& Nakayama, K. (2011). Where cognitive development and aging meet: Face learning ability peaks after age 30. Cognition, 118, 201-210. doi:https://doi.org/10.1016/j. cognition.2010.11.002

Germine, L., Duchaine, B., \& Nakayama, K. (2013). Face recognition memory data across the lifespan $N$ approx 60,000 . Retrieved from Figshare: doi:https://doi.org/10.6084/m9.figshare.767320.v1

Germine, L., Nakayama, K., Duchaine, B. C., Chabris, C. F., Chatterjee, G., \& Wilmer, J. B. (2012). Is the Web as good as the lab? Comparable performance from Web and lab in cognitive/ perceptual experiments. Psychonomic Bulletin \& Review, 19, 847 857. doi:https://doi.org/10.3758/s13423-012-0296-9

Gray, K. L. H., Bird, G., \& Cook, R. (2017). Robust associations between the 20-item prosopagnosia index and the Cambridge Face Memory Test in the general population. Royal Society Open Science, 4, 160923. doi:https://doi.org/10.1098/rsos.160923

Green, D. M., \& Swets, J. A. (1966). Signal detection theory and psychophysics. New York, NY: Wiley.

Kennerknecht, I., Grueter, T., Welling, B., Wentzek, S., Horst, J., Edwards, S., \& Grueter, M. (2006). First report of prevalence of non-syndromic hereditary prosopagnosia (HPA). American Journal of Medical Genetics, 140A, 1617-1622. doi:https://doi. org/10.1002/ajmg.a.31343

Kennerknecht, I., Nga, Y. H., \& Wong, V. C. N. (2008a). Prevalence of hereditary prosopagnosia (HPA) in Hong Kong Chinese population. American Journal of Medical Genetics, Part A, 146, 2863-2870. doi:https://doi.org/10.1002/ajmg.a.32552

Kennerknecht, I., Pluempe, N., \& Welling, B. (2008b). Congenital prosopagnosia - A common hereditary cognitive dysfunction in humans. Frontiers in Bioscience, 13, 3150-3158.

Kennerknecht, I., Plümpe, N., Edwards, S., \& Raman, R. (2007). Hereditary prosopagnosia (HPA): The first report outside the Caucasian population. Journal of Human Genetics, 52, 230-236. doi:https://doi.org/10.1007/s10038-006-0101-6

Livingston, L. A., \& Shah, P. (2017). People with and without prosopagnosia have insight into their face recognition ability. Quarterly Journal of Experimental Psychology, 71, 1260-1262. doi:https://doi.org/10.1080/17470218.2017.1310911

Malpass, R. S., \& Kravitz, J. (1969). Recognition for faces of own and other race. Journal of Personality and Social Psychology, 13, 330 334. doi:https://doi.org/10.1037/h0028434

Meissner, C. A., \& Brigham, J. C. (2001). Thirty years of investigating the own-race bias in memory for faces: A meta-analytic review. Psychology, Public Policy, and Law, 7, 3-35. doi:https://doi.org/ 10.1037/1076-8971.7.1.3

Palermo, R., Rossion, B., Rhodes, G., Laguesse, R., Tez, T., Hall, B., . . . McKone, E. (2017). Do people have insight into their face recognition abilities? Quarterly Journal of Experimental Psychology, 70, 218-233. doi:https://doi.org/10.1080/17470218.2016.1161058

Peterson, E., \& Miller, S. F. (2012). The eyes test as a measure of individual differences: How much of the variance reflects verbal IQ? Frontiers in Psychology, 3, 220. doi:https://doi.org/10.3389/fpsyg. 2012.00220

Rotshtein, P., Geng, J. J., Driver, J., \& Dolan, R. J. (2007). Role of features and second-order spatial relations in face discrimination, face recognition, and individual face skills: Behavioral and functional magnetic resonance imaging data. Journal of Cognitive Neuroscience, 19, 1435-1452. doi:https://doi.org/10.1162/jocn. 2007.19.9.1435

Shah, P., Gaule, A., Sowden, S., Bird, G., \& Cook, R. (2015a). The 20item prosopagnosia index (PI20): A self-report instrument for identifying developmental prosopagnosia. Royal Society Open Science, 2, 140343. doi:https://doi.org/10.1098/rsos.140343

Shah, P., Sowden, S., Gaule, A., Catmur, C., \& Bird, G. (2015b). The 20 item prosopagnosia index (PI20): Relationship with the Glasgow face-matching test. Royal Society Open Science, 2, 150305. doi: https://doi.org/10.1098/rsos.150305

Snowling, M., Dawes, P., Nash, H., \& Hulme, C. (2012). Validity of a protocol for adult self-report of dyslexia and related difficulties. Dyslexia, 18, 1-15. doi:https://doi.org/10.1002/dys.1432

Spackman, K. A. (1989). Signal detection theory: Valuable tools for evaluating inductive learning. In A. M. Segre (Ed.), Proceedings of the Sixth International Workshop on Machine Learning (pp. 160-163). San Mateo, CA: Morgan Kaufmann. doi:https://doi.org/10.1016/ B978-1-55860-036-2.50047-3

Stollhoff, R., Jost, J., Elze, T., \& Kennerknecht, I. (2011). Deficits in long-term recognition memory reveal dissociated subtypes in congenital prosopagnosia. PLoS ONE, 6, e15702. doi:https://doi.org/10. 1371/journal.pone.0015702

Turano, M. T., Marzi, T., \& Viggiano, M. P. (2016). Individual differences in face processing captured by ERPs. International Journal of Psychophysiology, 101, 1-8. doi:https://doi.org/10.1016/j.ijpsycho. 2015.12.009

Turano, M. T., \& Viggiano, M. P. (2017). The relationship between face recognition ability and socioemotional functioning throughout adulthood. Aging, Neuropsychology, and Cognition, 24, 613-630. doi:https://doi.org/10.1080/13825585.2016.1244247

White, D., Kemp, R. I., Jenkins, R., Matheson, M., \& Burton, A. M. (2014). Passport officers' errors in face matching. PLoS ONE, 9, e103510. doi:https://doi.org/10.1371/journal.pone.0103510

Wilmer, J. B. (2017). Individual differences in face recognition: A decade of discovery. Current Directions in Psychological Science, 26, 225 230. doi:https://doi.org/10.1177/0963721417710693

Wilmer, J. B., Germine, L., Chabris, C. F., Chatterjee, G., Gerbasi, M., \& Nakayama, K. (2012). Capturing specific abilities as a window into human individuality: The example of face recognition. Cognitive Neuropsychology, 29, 360-392. doi:https://doi.org/10.1080/ 02643294.2012 .753433

Wilmer, J. B., Germine, L., Chabris, C. F., Chatterjee, G., Williams, M., Loken, E., . . . Duchaine, B. (2010). Human face recognition ability is specific and highly heritable. Proceedings of the National Academy of Sciences, 107, 5238-5241. doi:https://doi.org/10. 1073/pnas.0913053107

Wilmer, J. B., Germine, L. T., \& Nakayama, K. (2014). Face recognition: A model specific ability. Frontiers in Human Neuroscience, 8, 769. doi:https://doi.org/10.3389/fnhum.2014.00769

Wilson, E. B. (1927). Probable inference, the law of succession, and statistical inference. Journal of the American Statistical Association, 22, 209-212. doi:https://doi.org/10.1080/01621459. 1927.10502953

Winblad, B., Palmer, K., Kivipelto, M., Jelic, V., Fratiglioni, L., Wahlund, L.-O., . . . Petersen, R. C. (2004). Mild cognitive impairmentBeyond controversies, towards a consensus: Report of the International Working Group on Mild Cognitive Impairment. Journal of Internal Medicine, 256, 240-246. doi:https://doi.org/10. $1111 /$ j.1365-2796.2004.01380.x

Youden, W. J. (1950). Index for rating diagnostic tests. Cancer, 3, 32-35. doi:https://doi.org/10.1002/1097-0142(1950)3:1<32::AIDCNCR2820030106>3.0.CO;2-3 